\title{
Pulmonary tuberculosis presenting with aortic pseudoaneurysm, diffuse alveolar hemorrhage, and cardiomyopathy: Is there an intriguing relationship?*
}

\author{
Min Shen ${ }^{1}$, Xuejun Zeng ${ }^{2 \#}$, Xingrong Liu ${ }^{3}$, Bao Liu ${ }^{4}$, Baotong Zhou ${ }^{5}$, Xue Lin $^{6}$, Ran Tian $^{6}$ \\ ${ }^{1}$ Department of Rheumatology, Peking Union Medical College Hospital (PUMCH), Chinese Academy of Medical Sciences (CAMS) \\ \& Peking Union Medical College (PUMC), Key Laboratory of Rheumatology and Clinical Immunology, Ministry of Education, \\ Beijing, China \\ ${ }^{2}$ Department of General Internal Medicine, PUMCH, CAMS \& PUMC, Beijing, China; \\ \#Corresponding Author: zxuejun@yahoo.com \\ ${ }^{3}$ Department of Cardiac Surgery, PUMCH, CAMS \& PUMC, Beijing, China \\ ${ }^{4}$ Department of Vascular Surgery, PUMCH, CAMS \& PUMC, Beijing, China \\ ${ }^{5}$ Department of Infectious Diseases, PUMCH, CAMS \& PUMC, Beijing, China \\ ${ }^{6}$ Department of Cardiology, PUMCH, CAMS \& PUMC, Beijing, China
}

Received 29 October 2013; revised 27 November 2013; accepted 10 December 2013

Copyright (C) 2013 Min Shen et al. This is an open access article distributed under the Creative Commons Attribution License, which permits unrestricted use, distribution, and reproduction in any medium, provided the original work is properly cited. In accordance of the Creative Commons Attribution License all Copyrights (C) 2013 are reserved for SCIRP and the owner of the intellectual property Min Shen et al. All Copyright (C) 2013 are guarded by law and by SCIRP as a guardian.

\begin{abstract}
A 22-year-old Chinese man presented with fever, cough, hoarseness, neck pain, acute heart failure and hemoptysis. Pulmonary tuberculosis was proved by sputum culture. Chest imaging showed an aortic arch pseudoaneurysm and bilateral ground glass opacities. Echocardiography confirmed cardiomyopathy. With antiTB drugs, high-dose prednisone and surgery, the life of this patient was successfully prolonged for more than four months. The concomitant disorders of aortic pseudoaneurysm, alveolar hemorrhage and cardiomyopathy in pulmonary tuberculosis are intriguing. We postulate that immune-mediated small vessel vasculitis triggered by pathogens plays an important role in the pathogenesis of this disease, rather than a direct TB infection.
\end{abstract}

Keywords: Pseudoaneurysm; Vasculitis; Thoracic Aorta

\section{INTRODUCTION}

Tuberculous pseudoaneurysm is a rare but fatal condition that is invariably indicative of disseminated tuber-

${ }^{*}$ Competing interests: The authors declare that they have no competing interests. culosis (TB), which is usually diagnosed with evidence of TB infection at other positions and fever and persistent pain, besides para-aortic mass [1]. TB is rarely associated to diffuse alveolar hemorrhage (DAH), and to our knowledge, pulmonary TB has been reported only twice as the cause of DAH [2].

Here, we report a culture-proven pulmonary TB patient concomitantly presenting with aortic pseudoaneurysm, DAH and cardiomyopathy, which has not been previously reported. The intriguing relationship among pseudoaneurysm, DAH, cardiomyopathy and TB will be discussed in this report.

\section{CASE PRESENTATION}

A 22-year-old Chinese man was admitted to our hospital, complaining of fever and cough for more than one month, aggravated in the recent 10 days. He also had fatigue, anorexia, and weight loss, without night sweat, rash, or arthralgia. Ten days before admission, he began to suffer from lower limb pain, and was unable to walk. He experienced progressive shortness of breath, hoarseness, cough productive of blood-tinged sputum, palpitation, oppressive pain of left side of the neck, and left upper extremity weakness. He was healthy before this admission. One of his classmates was diagnosed with pulmonary TB previous year. His mother had a history of pulmonary TB when she was five. He denied smoking and drug abuse. 
On admission he looked distressed, with a heart rate of $128 \mathrm{bpm}$, bilateral blood pressure of $140 / 82 \mathrm{~mm} \mathrm{Hg}$, and oxygen saturation of $92 \%$ under normal conditions. Physical examination revealed diminished breath sounds and rales in the right lower lung. Neurological examination was normal. Laboratory results showed a WBC count of $5.3 \times 10^{9} / \mathrm{L}\left(4.0-10.0 \times 10^{9} / \mathrm{L}\right)$, hemoglobin $126 \mathrm{~g} / \mathrm{L}(120-160 \mathrm{~g} / \mathrm{L})$, and platelet count of $374 \times$ $10^{9} / \mathrm{L}\left(100-300 \times 10^{9} / \mathrm{L}\right)$. Urinalysis and renal, hepatic, and coagulation profiles were normal. Serum investigations found a raised erythrocyte sedimentation rate (ESR) and C-reactive protein (CRP). Antinuclear antibodies, antiphospholipid antibodies and antineutrophil cytoplasmic antibodies were negative. He tested negative for the purified protein derivative of tuberculin (PPD), but his results of Mycobacterium tuberculosis (MTB)-specific IFN- $\gamma$ release assays were as high as $3620 \mathrm{SFCs} / 106$ PBMC. Bronchoscopy was conducted with normal findings. Multiple sputum sample smears found no evidence for bacterial, mycobacterial, or fungal organisms.

His condition worsened at night on the admission day, and he presented with severe cough, and orthopnea with hemoptysis. His pulse rate was $170 / \mathrm{min}$, blood pressure $150 / 100 \mathrm{~mm} \mathrm{Hg}$, respiratory rate $35 / \mathrm{min}$, and his oxygen saturation was only $90 \%$ under reservoir oxygen mask with velocity of flow $10 \mathrm{~L} / \mathrm{min}$. Chest examination revealed widespread rales of bilateral lungs. Laboratory testing showed an obvious drop of hemoglobin level from $126 \mathrm{~g} / \mathrm{L}$ to $101 \mathrm{~g} / \mathrm{L}$, and elevation of brain natriuretic peptide (BNP) $(1441 \mathrm{ng} / \mathrm{L}$; reference value 0 - 100 ng/L), while cardiac enzymes were normal. Chest CT scan (Figure 1) showed an aortic arch pseudoaneurysm with a $1.2 \times 3.0 \mathrm{~cm}$ defect in the lateral wall and mural thrombus. Meanwhile, high-resolution CT revealed bilateral areas of ground glass opacities. Echocardiography showed a decreased cardiac systolic function, suggestive of cardiomyopathy with an ejection fraction of only $48 \%$.

Sputum cultures grew Mycobacterium tuberculosis after his admission. The patient was treated with a standard therapy with rifampicin, isoniazid, ethambutol, pyrazinamide, and moxifloxacin. In addition, corticosteroids were given on the same day because vasculitis was suspected. His symptoms resolved in the first week while chest CT cleared in about 10 days (Figure 1). His left ventricular ejection fraction increased to $60 \%$. Two weeks later, he suddenly developed severe pain in the chest and lower extremities with high blood pressure, while the blood pressure of lower limbs could not be measured. Repeat CT revealed a further increase in the size of the pseudoaneurysm (Figure 1). The patient underwent an emergency surgery, with exclusion of the pseudoaneurysm. The pathology of the tissue of the aortic pseudoaneurysm revealed a creeping rupture of the media of the wall, and focal inflammatory cell infiltra-

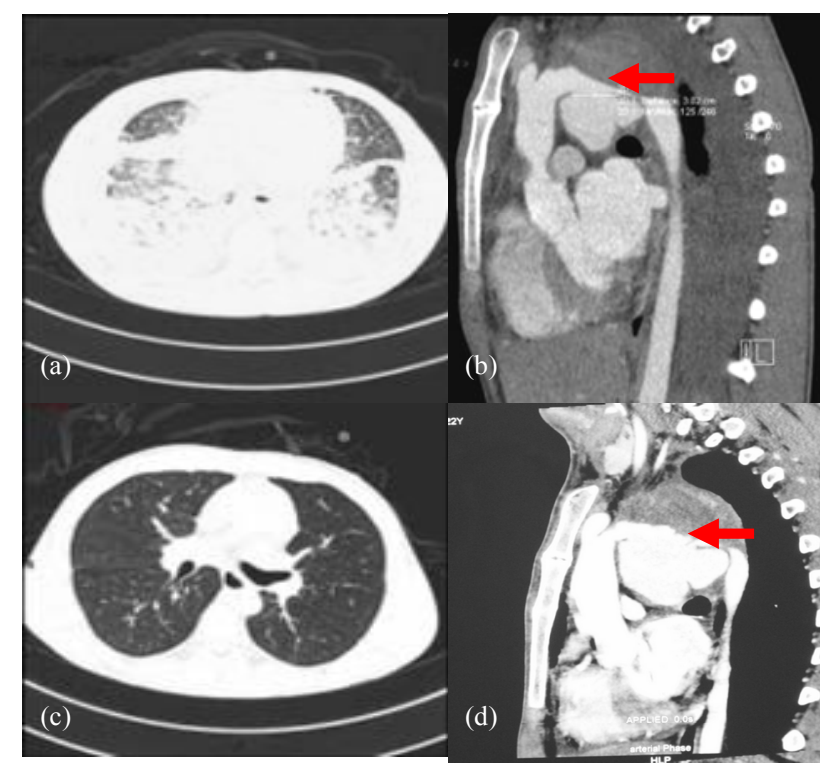

Figure 1. (a) High-resolution CT reveals bilateral ground glass opacities and pleural effusion. (b) Longitudinal contrast-enhanced CT scan shows a pseudoaneurysm arising from the aortic arch, with mural thrombus. CT scan also shows a $1.2 \times$ $3.0 \mathrm{~cm}$ defect in the lateral wall of the aortic arch (arrow), and the pseudoaneurysm compresses the adjacent aortic arch, left pulmonary artery, and left main bronchus. (c) High-resolution CT reveals that the lungs get clear after 10 days therapy of antiTB drugs and high-dose steroids. (d) Longitudinal contrastenhanced CT scan performed three weeks later (after image shown in A) reveals a further increase in the size of the pseudoaneurysm, with the defect in the lateral wall of the aortic arch increasing to $1.5 \times 3.7 \mathrm{~cm}$ (arrow).

tion at the adventitia, without caseous necrosis. In addition, the culture of the tissue was negative for any organisms. The anti-tubercular chemotherapy was continued, with corticosteroids tapered gradually. After 19 weeks of the operation, the patient had sudden onset hematemesis and hypovolemic shock, and died soon thereafter. The postmortem could not be performed.

\section{DISCUSSION}

A patient who developed aortic pseudoaneurysm, $\mathrm{DAH}$, cardiomyopathy, and pulmonary $\mathrm{TB}$ is described in this report. Other infectious, malignant, or immunological etiologies for the development of aortic pseudoaneurysm, DAH, and cardiomyopathy were excluded after extensive studies. We therefore think that these disorders were the complications of TB infection. The concomitant appearance of all the unusual complications in one patient has not been reported in literature.

So far, TB as the cause of pseudoaneurysm has been only occasionally reported, with less than 50 cases since 1945 in the literature in English [1,3-7]. Typical clinical scenarios of tuberculous aortic pseudoaneurysm include evidence of TB infection elsewhere in the body, with 1 or 
more of 3 presentations: fever and persistent pain related to the location of the pseudoaneurysm, dysphagia, or hoarseness; evidence of massive bleeding; or pulsative, rapidly expanding para-aortic mass [1]. In our patient, pseudoaneurysm with pulmonary $\mathrm{TB}$ should raise the suspicion of tuberculous aortic pseudoaneurysm. But our case was unusual because neither anti-fast bacilli nor caseous necrosis was detected from the tissue of the aortic pseudoaneurysm. Therefore, the cause of pseudoaneurysm in our patient might have been secondary to vasculitis which was strongly related to the TB infection, as could be seen in the relation between TB and Takayasu arteritis [8], or that between TB and Behcet disease [9].

In our patient, the diagnosis of DAH relied on clinical (hemoptysis and dyspnea), laboratory (rapid drop of hemoglobin), and typical radiological findings. The possible mechanisms for TB associated DAH include infection related diffuse alveolar damage, immune-mediated process, and antiphospholipid antibodies [2,10]. Coincidentally, cardiomyopathy was also confirmed by echocardiography. The causal link between TB, DAH and cardiomyopathy was strongly supported by the prompt clinical response to anti-TB therapy and corticosteroids. Indeed, the clinical outcomes claimed that immune-mediated damage caused by TB infection might have played an important role in the pathogenesis of DAH and cardiac involvement in this patient.

Considering the above findings, aortic pseudoaneurysm, DAH and cardiomyopathy in this TB patient were most likely caused by immune-mediated vasculitis rather than direct TB infection, though we did not find a firsthand evidence of vasculitis. The patient should have been treated with anti-TB drugs and steroids, coupled with surgery as soon as possible. Unfortunately, the case was complicated with DAH and cardiomyopathy at the time of admission, because of which definitive surgical treatment could not be instituted on time. Emergency operation thereafter helped extend his life for more than four months, but he eventually expired, most probably owing to the perforation contained in the exclusion pseudoaneurysm into the gastrointestinal tract.

To our knowledge, this is the first reported case of active pulmonary TB complicated with aortic aneurysm, $\mathrm{DAH}$, and cardiomyopathy in an immune-competent patient. However, because of a lack of previously published experience on diagnosis and therapy, we were confronted with enormous difficulties. With prompt commencement of anti-TB therapy, high-dose prednisone, and surgical treatment, it is possible to offer the best chance for a cure and constitute the only way to salvage patients struck with this disease. Medical management alone or surgical management alone is not adequate. As the prevalence of TB is rising worldwide, we hope this report will increase alertness in physicians to the com- plications of TB infection.

\section{CONSENT}

Written informed consent was obtained from the families of the patient for publication of this case report and accompanying images. A copy of the written consent is available for review by the Editor-in-Chief of this journal.

\section{AUTHORS' CONTRIBUTIONS}

MS was involved in the conception of the report, literature review, manuscript writing and submission. $\mathrm{XZ}$ was involved in the conception of the report, manuscript critique and review. XL and BL performed the surgery and involved in the manuscript critique and review. BZ, $\mathrm{XL}$ and RT were involved in the diagnosis and treatment course, manuscript critique and review. All authors read and approved the final manuscript.

\section{ACKNOWLEDGEMENTS}

This work was supported by the Clinical Research Funds of Chinese Medical Association (grant number 12040760376), and the Youth Research Funds of Peking Union Medical College Hospital (grant number 2010103).

\section{REFERENCES}

[1] Kon, A.S., Grumach, A.S., Colombo, A.L., et al, (2008) Guidelines in cryptococcosis. Revista da Sociedade Brasileira de Medicina Tropical, 41, 524-544.

[2] Patz Jr., E.F. and Goodman, P.C. (1992) Pulmonary cryptococcosis. Journal of Thoracic Imaging, 7, 51-55. http://dx.doi.org/10.1097/00005382-199209000-00008

[3] Severo, C.B., Gazzoni, A.F. and Severo, L.C. (2009) Pulmonary cryptococcosis. Jornal Brasileiro de Pneumologia, 35, 1136-1144. http://dx.doi.org/10.1590/S1806-37132009001100012

[4] Silva, A.C.G., Marchiori, E., Souza Jr., A. and Irion, K.L. (2003) Criptococose pulmonar: Aspectos na tomografia computadorizada. Radiologia Brasileira, 36, 277-282. http://dx.doi.org/10.1590/S0100-39842003000500005

[5] Guimarães, M.D., Marchiori, E., Meirelles, G.S.P., et al. (2013) Fungal infection mimicking pulmonary malignnancy: Clinical and radiological characteristics. Lung, 191, 655-662. http://dx.doi.org/10.1007/s00408-013-9506-0

[6] Fong, I.A., Glória, C., Mesquita, I. and Diogo, N. (1999) Pulmonary cryptococcosis in an immunocompetent patient. A case report and a review. Revista Portuguesa de Pneumologia, 4, 419-426.

[7] Barbosa, A.T.F., Colares, F.A., Gusmão, E.S., Barros, A.A., Cordeiro, C.G. and Andrade, M.C.T. (2006) Isolated pulmonary cryptococcosis in an immunocompetent patient. Jornal Brasileiro de Pneumologia, 32, 470-480. http://dx.doi.org/10.1590/S1806-37132006000500016 


\section{LIST OF ABBREVIATIONS}

DAH: diffuse alveolar hemorrhage; TB: tuberculosis;
ESR: erythrocyte sedimentation rate; CRP: C-reactive protein; BNP: brain natriuretic peptide. 